\title{
Managing coverage holes in IoT monitoring sensor networks
}

\author{
Roya Habibi ${ }^{\text {a }}$, Ali Asghar Alesheikh ${ }^{\text {a, * }}$ \\ ${ }^{a}$ Faculty of Geodesy and Geomatics Engineering, K. N. Toosi University of Technology, Tehran, Iran, rhabibi@mail.kntu.ac.ir, \\ alesheikh@kntu.ac.ir \\ * Corresponding author
}

Keywords: Internet of Things, Environmental Monitoring, Interpolation, Time Series Analysis

\begin{abstract}
:
Coinciding with the development of sensor networks, communication technologies and also emerging the Internet of Things (IoT), the way was smoothed for the improvement of environmental observation tasks. The basic considered architecture for IoT is a 3-layer architecture including application, network, and perception layers respectively. The lowest layer is tasked with sensing the physical world. In environmental observation and monitoring, in-situ sensing and remote sensing generally do this duty. If the variability of the phenomenon in space and time is high such as air pollution concentrations, in-situ sensing is the best solution. Gathered data from physical world deliver to application layer through network layer. Services and analyses of monitoring tasks offer and conduct in application layer.

There are lots of researches in the literature which have addressed requirements and challenges of IoT including network architecture and infrastructure [1], security and privacy [2], big data analytics [3]. Natural phenomena are inherently continuous. Ideally, in IoT scenarios, it is needed to know observing phenomenon at anytime and anywhere. At this point, sensor measurement rate and spatial coverage of sensor network are problematic. This is an undeniable fact that the phenomenon cannot be sensed at any place. Most sensors are statics. In addition, setup and maintenance of them are expensive. Therefore, data of interested phenomenon is measured at a number of distinct points. Node failure is one of the critical issues in wireless sensor networks which leads to a coverage hole. These holes in sensor networks result in the degradation of network performance and quality of service [4]. However, it might be a minor problem depending on spatial variability of observing phenomenon or sensor network density. Spatial interpolation methods are the best keys to solve this problem. In this way, a continuous surface of interested parameter is estimated throughout the area and values for unmeasured points are predicted. According to the first low of geography, there is a spatial relation between spatial features in geographic space. Spatial autocorrelation is a characteristic implying this relation and define a distance in which observed values are correlated with itself [5]. This correlation might be positive or negative. Nonetheless, it is meaningful at a range. This range is essential in monitoring task. It plays a prominent role in sensor network density. If sensor network structure is so dense that cover out of range distances according to spatial autocorrelation, this surface will be acceptably precise. If a node failure happen in monitoring network, precision of resulted interpolated surface will decrease and predicted values at unobserved points will be unreliable. The main purpose of the current work is providing a solution to manage this coverage hole in IoT scenarios with earth observation tasks. In other words, it makes concentration values of pollutants available at right place and right time via web.
\end{abstract}

The case study for implementing solution is air pollution monitoring network in Tehran, Iran. Air pollution is one of the main problems in cities, especially in metropolises. In this regard, Tehran is faced with serious matters, and damages caused by this issue inflicts exorbitant costs on urban residents and officials. High intrinsic dynamic of air pollution in space and time, and contributions of natural or synthetic sources to its increase or reduction, clarify the necessity of observation systems which should be adopt to this dynamic. To overcome node failure in network, short-term time series analysis methods are promising. A time series is a sequence of observations that occur continuously in time [6]. The complexity of the physical and chemical processes that lead to changes in concentrations of atmospheric pollutants, and the complexity of geographical conditions, along with the presence of data errors in involving parameters, cause serious problems in precise and rapid modelling of pollutants. Therefore, for practical purposes, short-term predictive methods for concentrations of air pollutants are mainly based on time series statistical methods [7]. Using Holt-Winters temporal model, a method is proposed for covering missed data among observations of sensor network. This study was performed on air quality monitoring network of Tehran and utilized OGC SWE standards to produce interoperable open spatial infrastructure in sensor networks. For performing statistical tests, two stations named Cheshmeh and Elm-oSanat were detected as samples of clean and polluted regions using Moran's I index spatial clustering method. For spatial prediction of pollutant, various kind of spatial interpolation methods will be evaluated and best method will be selected due to less error in fitting a surface of pollutants throughout the city. Finally, the desired models will be integrated in a data infrastructure established by sensor web standards and results will be available for users through a web-based application. These results could be usable in other applications of internet of things such as related tasks to public health, gathering correct and timely decisions by city policy-makers during the advent of air pollution critical conditions and reduction or prevention of it. 
1. Schulz, P.; Matthe, M.; Klessig, H.; Simsek, M.; Fettweis, G.; Ansari, J.; Ashraf, S.A.; Almeroth, B.; Voigt, J.; Riedel, I. Latency critical iot applications in $5 \mathrm{~g}$ : Perspective on the design of radio interface and network architecture. IEEE Communications Magazine 2017, 55, 70-78.

2. Zhou, J.; Cao, Z.; Dong, X.; Vasilakos, A.V. Security and privacy for cloud-based iot: Challenges. IEEE Communications Magazine 2017, 55, 26-33.

3. Cai, H.; Xu, B.; Jiang, L.; Vasilakos, A.V. Iot-based big data storage systems in cloud computing: Perspectives and challenges. IEEE Internet of Things Journal 2017, 4, 75-87.

4. Yi, L.; Deng, X.; Wang, M.; Ding, D.; Wang, Y. Localized confident information coverage hole detection in internet of things for radioactive pollution monitoring. IEEE Access 2017, 5, 18665-18674.

5. Habibi, R.; Alesheikh, A.; Mohammadinia, A.; Sharif, M. An assessment of spatial pattern characterization of air pollution: A case study of co and pm2. 5 in tehran, iran. ISPRS International Journal of Geo-Information 2017, 6, 270.

6. Box, G.E.; Jenkins, G.M.; Reinsel, G.C.; Ljung, G.M. Time series analysis: Forecasting and control. John Wiley \& Sons: 2015.

7. Kumar, U.; Jain, V. Arima forecasting of ambient air pollutants (o3, no, no2 and co). Stochastic Environmental Research and Risk Assessment 2010, 24, 751-760. 